\title{
A Theoretical and Experimental Study of Electrochemical pH Control at Gold Interdigitated Microband Arrays
}

Benjamin O’Sullivan ${ }^{\mathrm{a}}$, Bernardo Patella ${ }^{\mathrm{a}, \mathrm{b}}$, Robert Daly ${ }^{\mathrm{a}}$, Ian Seymour ${ }^{\mathrm{a}, \mathrm{c}}$, Pierre Lovera ${ }^{\mathrm{a}}$, James F. Rohan ${ }^{\mathrm{c}}$, Rosalinda Inguanta $^{\mathrm{b}}$, Alan O’Riordan ${ }^{\mathrm{a}}$

a Nanotechnology Group, Tyndall National Institute, University College Cork, Cork T12 R5CP, Ireland

b Laboratorio di Chimica Fisica Applicata, Dipartimento di Ingegneria, Università of Palermo, Viale delle Scienze, Palermo, Italy

c Electrochemical Materials and Energy Group, Tyndall National Institute, University College Cork, Cork T12 R5CP, Ireland

\begin{abstract}
In electroanalysis, solution $\mathrm{pH}$ is a critical parameter that often needs to be adjusted and controlled for the detection of particular analytes. This is most commonly performed by the addition of chemicals, such as strong acids or bases. Electrochemical in-situ $\mathrm{pH}$ control offers the possibility for the local adjustment of $\mathrm{pH}$ at the point of detection, without additional reagents. FEA simulations have been performed to guide experimental design for both electroanalysis and in-situ control of solution $\mathrm{pH}$. No previous model exists that describes the generation of protons at an interdigitated electrode array in buffered solution with one comb acting as a protonator, and the other as the sensor. In this work, FEA models are developed to provide insight into the optimum conditions necessary for electrochemical $\mathrm{pH}$ control. The magnitude of applied galvanostatic current has a direct relation to the flux of protons generated and subsequent change in $\mathrm{pH}$. Increasing the separation between the electrodes increases the time taken for protons to diffuse across the gap. The final $\mathrm{pH}$ achieved at both, protonators and sensor electrodes, after 1 second, was shown to be largely uninfluenced by the initial $\mathrm{pH}$ of the solution. The impact of buffer concentration was modelled and investigated. In practice, the $\mathrm{pH}$ at the electrode surface was probed by means of cyclic voltammetry, i.e., by cycling a gold electrode in solution and identifying the potential of the gold oxide reduction peak. A pH indicator, methyl red, was used to visualise the solution $\mathrm{pH}$ change at the electrodes, comparing well with the model's prediction.
\end{abstract}

\section{Keywords}

Finite element analysis simulation, in-situ pH control, solid state sensors, Electroanalysis, Microband interdigitated electrode $\operatorname{array}(6 \max )$ 


\section{Introduction}

The $\mathrm{pH}$ of a solution is an important parameter in many biological and chemical reactions, either as a catalyst or where the protons are active as reactant or product. The detection of numerous analytes, including porphyrins[1], nitrates/nitrites[2, 3], chlorine[4] and human haemoglobin[5], requires the adjustment of $\mathrm{pH}$ from neutral to acidic conditions. The typical method of changing solution $\mathrm{pH}$ is the rudimentary addition of buffers or strong acids and bases. A number of issues can arise from this method including the purchase of additional chemicals, the requirement for trained personnel to handle them, the added safety risk and finally the potential to act as a source of contamination. Controlling the $\mathrm{pH}$ by in-situ electrochemical methods allows the user to adjust the $\mathrm{pH}$ both temporally and spatially and perform electroanalysis with the same device; thereby eliminating the extra steps. Potentially important electrochemical $\mathrm{pH}$ control methods include electrochemical redox reactions[6] and water electrolysis[7]. The latter is the splitting of water with an electrical current and can be used to reduce the solution $\mathrm{pH}$ by applying a positive potential to consumes hydroxyl ions in a basic solution or produce protons in acidic conditions.

Finite element analysis (FEA) simulations have been used to provide valuable insight into a variety of electrochemical processes. Mass transport at electrode arrays has been modelled to determine inter-electrode spacing needed to achieve diffusional independence $[8,9]$. The simulations directly informed the subsequent sensor design and fabrication. Similarly, the theoretical study of ionic transfer at liquid- liquid interfaces has guided the development of micro and nano-ITIES (interface between two immiscible electrolyte solutions) systems[10]. Adsorption processes at electrode surfaces have also been simulated, to assess reactions taking place at the electrode as the electrode itself is modified[11]. Wahl et al. demonstrated the use of simulations for iron detection at interdigitated microband electrodes(IDE) and the expected collection efficiency in generatorcollector(GC) mode[12]. The spacing between the electrode combs is inversely proportional to the diffusional overlap and thus, at smaller inter-comb distances a greater degree of overlap is present.

The modelling of solution $\mathrm{pH}$ change and its effects has also been used to inform on experimental design and comparison to results. Read et al[13] modelled the change in $\mathrm{pH}$ at concentric boron doped diamond ring electrodes. Protons were generated at the outer electrode and the decreased local $\mathrm{pH}$ was monitored by use of an iridium oxide electrode. While elegant, this model did not account for a solution that contained buffering agents. Critelli et al[14] have shown the effect of buffer reacting with generated protons and the impact on the rate of solution $\mathrm{pH}$ change. Proton flux, buffering effects and competing simultaneous chemical reactions were all investigated. While this model focused on the effects of probing the $\mathrm{pH}$ near the electrode surface, similar equations are applicable to models focused on directly changing the local $\mathrm{pH}$. The combination of both models would allow the simulation of proton flux generated by a fixed current and the subsequent buffering effects to be accounted for. 
In this work, FEA simulations are performed for proton generation by water electrolysis. This approach builds upon the works of Read and Critelli mentioned above by modelling the generation of protons at one comb of interdigitated electrodes and their buffered diffusion towards the other comb and towards the bulk solution. A galvanostatic current is applied at one array, with the flux of protons proportional to the applied current. The importance of interelectrode distance is investigated by modelling the proton generation at 3 different electrode separations. In response to these simulations, gold interdigitated microband arrays are designed and fabricated for the monitoring and control of solution $\mathrm{pH}$. Solution buffer capacity was also investigated as it can impact on the change in $\mathrm{pH}$ at the surface of the sensing electrodes. In our approach, the gold oxide reduction peak is used as a probe for local solution $\mathrm{pH}$ at the working electrodes across a range of $\mathrm{pH}$ buffers. The change in solution $\mathrm{pH}$ by electrochemical control at the electrode array is visualized optically by use of a $\mathrm{pH}$ indicator, methyl red. The region of colour change is compared to the simulated proton concentration profiles.

\section{Materials and Methods}

\subsection{Materials and Electrode Characterization}

Nitric acid (35\%), sodium chloride, ferrocene carboxylic acid (FcCOOH), phosphate buffer solution (PBS), copper chloride, disodium phosphate and citric acid were purchased from Sigma Aldrich, Ireland and used as received. All solutions were prepared with DI water with resistivity of $18 \mathrm{M} \Omega \mathrm{cm}^{-1}$. A CHI 920 bi-potentiostat was used for the analytical measurements with the images captured on a Zeiss microscope equipped with a charge-coupled detector camera(CCD). All experiments were undertaken in a four electrode cell comprising of the on chip gold counter electrode and either an $\mathrm{Ag} / \mathrm{AgCl}$ as external reference electrode or the on chip platinum pseudo reference electrode. The interdigitated working electrodes were electrochemically characterized by cyclic voltammetry using $1 \mathrm{mM} \mathrm{FcCOOH}$ in $10 \mathrm{mM}$ PBS pH 7.4 solution. The potential was cycled between $-0.15 \mathrm{~V}$ and $0.45 \mathrm{~V}$ vs. an on chip platinum pseudo-reference electrode with a scan rate of $100 \mathrm{mV} / \mathrm{s}$. Sensing electrodes of $1 \mu \mathrm{m}$ width with a $2 \mu \mathrm{m}$ gap to the protonators typically exhibited a plateau current of $10 \mathrm{nA}$ in non-GC mode, and $28 \mathrm{nA}$ in GC mode analysis.

\subsection{In-situ pH control simulation study}

Figure S1A shows a schematic of the in-situ $\mathrm{pH}$ control concept. GC electrode array devices composed of two combs of interdigitated electrodes were employed for in-situ $\mathrm{pH}$ control in this work. A comb, in this regard, refers to one half of an interdigitated electrode array. The 
interdigitated electrodes are spaced $2 \mu \mathrm{m}$ apart. By imposing an appropriate potential at one ("protonator") comb of electrodes, a $\mathrm{pH}$ change occurs in the local environment that tailors the $\mathrm{pH}$ at the other ("sensor") comb. The sensor comb may then be used to perform sensing in conditions that differ from the bulk solution. In our approach, hydrolysis of water is employed to produce protons at the protonator electrode, which then diffuse to and lower the $\mathrm{pH}$ in the region of the sensor electrode; compared to the bulk solution.[13]The $\mathrm{pH}$ may be tailored to the required value by control of the applied current density.[15] In acidic conditions, the $\mathrm{pH}$ can be changed through the water splitting reactions of equations 1-2:[16]

Cathode: $2 \mathrm{H}^{+}+2 e^{-} \rightarrow \mathrm{H}_{2}$

Anode: $2 \mathrm{H}_{2} \mathrm{O} \rightarrow \mathrm{O}_{2}+4 \mathrm{H}^{+}+4 e^{-}$

In basic conditions, the mechanism becomes:

$$
\begin{aligned}
& \text { Cathode: } 2 \mathrm{H}_{2} \mathrm{O}+2 e^{-} \rightarrow 2 \mathrm{OH}^{-}+\mathrm{H}_{2} \\
& \text { Anode: } 4 \mathrm{OH}^{-} \rightarrow \mathrm{O}_{2}+2 \mathrm{H}_{2} \mathrm{O}+4 e^{-}
\end{aligned}
$$

An acidic medium can be made more basic by consumption of protons in a reduction reaction, or alternatively more acidic by production of protons in an oxidation reaction (depending on the potential imposed at an electrode), and vice versa. It is important to note that the maximum $\mathrm{pH}$ changes occur close to the electrode and diminishes with increasing distance from the electrode.

A simulation model was designed to explore generation and diffusion of protons at the microband electrodes, using the FEA software COMSOL Multiphysics 5.3, in line with the galvanostatic model shown by Read et al[13]. The geometry of the model consisted of a $5 \mathrm{~mm}$ square box as the experimental domain, and two sets of interdigitated $1 \mu \mathrm{m}$ wide microband electrodes of $50 \mathrm{~nm}$ height (14 protonator electrodes and 13 sensing electrodes), separated by $2 \mu \mathrm{m}$; see Figure S1B and C. In the model, a flux of protons was applied to the surface of the protonators, by applying a fixed anodic current. The flux was assumed to be proportional to a galvanostatic current applied at the electrodes;

$$
F l u x=\frac{i}{F \cdot A}
$$

where "i" is the current applied, F is Faraday's constant and A is the surface area of the electrodes. The initial $\mathrm{pH}$ was set to $\mathrm{pH}$ 7. The proton diffusion coefficient used for the simulation was 9.31 $\mathrm{x} 10^{-5} \mathrm{~cm}^{2} \mathrm{~s}^{-1}$.[17] Diffusion of protons was modelled according to Fick's second law. The possible effects of alkalinity, electrode separation and buffering capacity on the diffusion profiles were 
investigated. The bulk $\mathrm{pH}$ modelled was from modelled $\mathrm{pH} 6$ to 10. Interdigitated electrode array separation of 1 and $10 \mu \mathrm{m}$ were also modelled.

The effects of a buffer were modelled by introduction of chemical equilibriums, as shown by Critelli et al [14]reflecting the reactions between the protons, the buffer molecule and water dissociation

$$
\begin{aligned}
& \mathrm{H}^{+}+\mathrm{OH}^{-} \rightarrow \mathrm{H}_{2} \mathrm{O} \quad k_{w}=-14 \\
& H^{+}+A^{-} \rightarrow H A \quad k_{a}=-6.8
\end{aligned}
$$

Ions $\mathrm{OH}^{-}, \mathrm{HA}$ and $\mathrm{A}^{-}$were defined, reflecting the hydroxyl ion, protonated and unprotonated buffer molecules, respectively. $\mathrm{k}_{\mathrm{w}}$ is the water dissociation constant, while $\mathrm{k}_{\mathrm{a}}$ is the acid dissociation constant. Initial concentration of the buffer was set at $1 \mathrm{mM}$ to reflect the concentration of sodium bicarbonate in the experimental buffer. The diffusion coefficients utilised were previously reported: proton $9.31 \times 10^{-5} \mathrm{~cm}^{2} \mathrm{~s}^{-1}$; hydroxyl $5.31 \times 10^{-5} \mathrm{~cm}^{2} \mathrm{~s}^{-1}$; protonated and unprotonated buffer $1 \times 10^{-5} \mathrm{~cm}^{2} \mathrm{~s}^{-1} .[14,17]$

Mesh quality is an important consideration when using FEA. As the model domain is divided into smaller sections or "elements", the collection of lines of division are known as a mesh. A key balance to meshing is the relationship between mesh quality and its computational time or cost. It is necessary for mesh quality to be high enough to obtain an accurate result, but a higher mesh quality results in more elements and thus a longer time to obtain a solution. Extra refinement is needed around the smaller features of the domain (the microbands) in comparison to the remainder of the domain. In these experiments, an error of $1 \%$ was targeted. The mesh refinement was acceptable once increasing the refinement no longer produced a discernible change on the concentration profiles.

\subsection{Electrode Fabrication}

Silicon chip sensor devices, consisting of interdigitated gold microband electrode arrays, a gold counter electrode and a platinum pseudo reference, were fabricated as described previously[12, 18]; see Figure S2A. In brief, interdigitated gold microbands were patterned in resist by photolithography followed by metal evaporation (Ti/Au 5/50 nm) and standard lift-off. Similarly, photolithography, metal evaporation (Ti/Au 10/90), and lift-off procedures were then employed to overlay electrical interconnection tracks including peripheral probe pads. Macroscale gold and platinum counter and pseudo-reference electrodes, respectively, was also deposited during this process. Finally, a silicon nitride passivation layer (500 nm thick) was deposited to passivate the 
entire chip and windows selectively opened with a dry etch to allow exclusive contact between the working, reference and counter electrodes with the solution of interest.

\subsection{Electrochemical approaches}

\section{Gold oxide reduction peak}

The effect of the solution $\mathrm{pH}$ on gold oxide reduction was studied. Gold oxide was formed and then reduced by cyclic voltammetry over a potential range of $-0.15 \mathrm{~V}$ to $1.2 \mathrm{~V}$ on a gold working electrode array at $100 \mathrm{mV} / \mathrm{s}$. The solution $\mathrm{pH}$ was varied chemically over a range of $\mathrm{pH} 3$ to 8 by preparing a series of buffer solutions of citric acid and disodium phosphate from $\mathrm{pH} 3$ to 8 . For electrochemical $\mathrm{pH}$ control, a series of potentials high enough to induce water electrolysis $(1.3 \mathrm{~V}$ to $1.55 \mathrm{~V}$ ) was applied on the protonator electrode array during the $\mathrm{CV}$.

\section{5. pH Change visualisation}

An artificial drinking water solution (ADW) containing the $\mathrm{pH}$ indicator, methyl red, was used to visualize the change in $\mathrm{pH}$ experimentally. ADW was made up by dissolving $1 \mathrm{~g}$ of sodium bicarbonate, $0.0654 \mathrm{~g}$ of magnesium sulfate (Sigma Aldrich, 99.5\% anhydrous), $0.3414 \mathrm{~g}$ calcium sulfate dehydrate (Honeywell, 99\%), $0.007 \mathrm{~g}$ potassium phosphate dibasic (Fluka, 98\%), potassium phosphate monobasic (Sigma Aldrich, 99\%) and $0.01 \mathrm{~g}$ sodium nitrate (Sigma Aldrich, 99\%) in 10 litres of deionized water. $50 \mathrm{mg}$ of methyl red was dissolved in $25 \mathrm{ml}$ of ethanol. This solution was then made up to $250 \mathrm{~mL}$ with $25 \mathrm{mM}$ ADW solution. A portable $\mathrm{CHI}$ $1222 \mathrm{c}$ potentiostat was used for visualization experiments. A current of $500 \mathrm{nA}$ was applied at the protonator. The images were captured on a Zeiss microscope equipped with a charge-coupled detector camera.

\section{Results and Discussion}

\subsection{Simulation of $\mathbf{p H}$ change vs Increasing Current}

FEA simulations were performed to study the generation and diffusion of protons at an interdigitated array of electrodes. The array studied consisted of interdigitated combs of $1 \mu \mathrm{m}$ wide electrodes (13 sensors, 14 protonators) of $50 \mathrm{~nm}$ height separated by $2 \mu \mathrm{m}$ in a $5 \mathrm{~mm}$ square domain. A flux of protons is generated at the protonator array (surface area of $15.4 \mu \mathrm{m}^{2}$ ) by applying a galvanostatic current. Figures 1A-D show proton concentration profiles as $\mathrm{pH}$ after 10 seconds of flux in a solution with starting $\mathrm{pH}$ of 7 (blue) over a range of applied currents. Figure 
1A, with a current of $1 \mathrm{nA}$, presents a semicircular change in colour $(\mathrm{pH})$ around the electrodes, with the largest colour change (red) closest to the electrodes. This change in colour represents the diffusion zone of the protons generated at the protonator array, with a greater $\mathrm{pH}$ change near the electrodes and gradually returning to bulk conditions at greater distances from the electrodes.

Figures 1B, C and D are the concentration profiles for applied currents of 10, 100 and $1000 \mathrm{nA}$, respectively, and each possess a diffusion zone with an increased diameter and a greater proportion of red ( $\mathrm{pH} 4$ and below) than the lower currents. From equation 5, a larger current generates a larger flux of protons and the resulting lower $\mathrm{pH}$. Figure $1 \mathrm{E}$ is a plot of solution $\mathrm{pH}$ at the electrode top surface $(50 \mathrm{~nm})$ across the width of the domain. A lower $\mathrm{pH}$ is reached with a larger current. At each applied current, there is a very sharp decrease in $\mathrm{pH}$ observed at the center of the domain (shown in Figure $1 \mathrm{E}$ at 2,500 $\mu \mathrm{m}$ on the $\mathrm{x}$-axis), with a more gradual change in $\mathrm{pH}$ moving from the center to the edges until bulk conditions again towards the edges. This enhanced drop in $\mathrm{pH}$ in the central regions (Figure 1E inset) occurs directly at the electrodes since the protonators are generating protons at a higher rate than they can diffuse away thereby creating a region of lower $\mathrm{pH}$ above the electrodes.
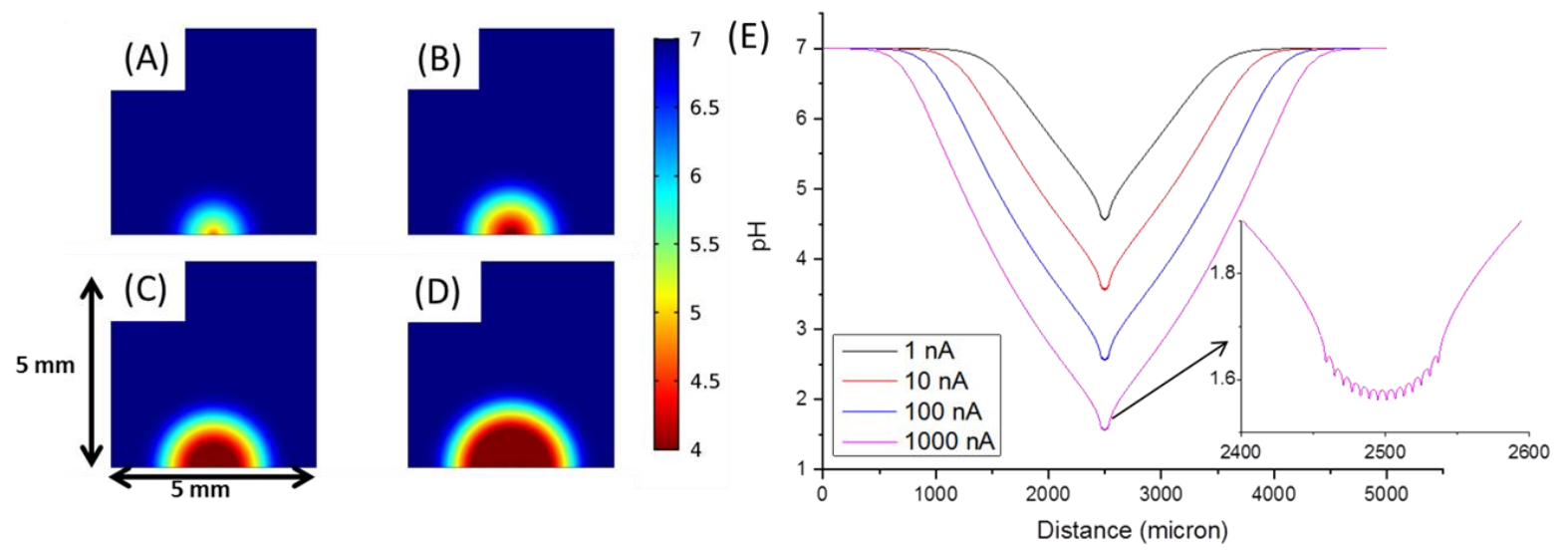

Figure 1Simulated pH profile after 10 seconds with applied current of 1 (A) , 10 (B), 100 (C) and 1000 (D) $\mathrm{nA}$ at protonators (Surface Area $15.4 \mu \mathrm{m}^{2}$ ); (E) Plot of $\mathrm{pH}$ at height over the electrodes vs width of model domain. Inset: Magnified image of region directly surrounding electrode array.

\subsection{Electrode Separations}

The effect of the changing the electrode separations in the interdigitated arrays viz $1 \mu \mathrm{m}, 2 \mu \mathrm{m}$ and $10 \mu \mathrm{m}$ between the protonator and sensing combs was investigated after 100 microseconds and 1 second of applied current of $100 \mathrm{nA}$ since increasing the duration of applied current should impact distance over which protons diffuse. We note that the $80 \mu \mathrm{m}$ passivation window, in which the array is located, limited the number of $1 \mu \mathrm{m}$ width microband electrodes that can be present as the spacing between combs increases. A $1 \mu \mathrm{m}$ separation array contained 20 protonators and 20 
sensors, a $2 \mu \mathrm{m}$ array contains 14 protonators and 13 sensors, a $10 \mu \mathrm{m}$ array contained 4 protonators and 4 sensors. Figure 2A top panel shows the proton concentration profile after 100 microseconds of the $10 \mu \mathrm{m}$ gap array. There are semicircular diffusion zones around each of the 4 protonator electrodes representing the change in $\mathrm{pH}$. The clear separation between the diffusion zones is demonstrated in Figure 2A bottom panel (black), where bulk solution pH is present at the sensing electrodes (the midpoint between protonators). Figure $2 \mathrm{~A}$ bottom panel (red) also displays the $\mathrm{pH}$ after 1 second which has decreased significantly. After this time, the zones of decreased $\mathrm{pH}$ around each of the 4 protonator electrodes have overlapped significantly, though we note there is a small deviation of $\sim 0.1 \mathrm{pH}$ units midpoint between protonators (over the sensing electrodes). Figure 2B top panel shows the proton concentration profile after 100 microseconds of the $2 \mu \mathrm{m}$ gap array. There are semicircular diffusion zones around each of the 14 protonator electrodes representing the change in $\mathrm{pH}$. The overlap of these individual diffusion zones is demonstrated in Figure 2B bottom panel (black), where bulk solution $\mathrm{pH}$ is not present at the sensing electrodes (the midpoint between protonators). Figure 5B bottom panel (red) shows that the $\mathrm{pH}$ variation midpoint between protonators (i.e., at the sensors), has decreased to $\sim 0.02 \mathrm{pH}$ units after 1 second. Figure $2 \mathrm{C}$ top panel, shows the proton concentration profile after 100 microseconds of the $1 \mu \mathrm{m}$ gap array. There are semicircular diffusion zones around each of the 20 protonator electrodes representing the change in $\mathrm{pH}$. The overlap of these individual diffusion zones is demonstrated in Figure $2 \mathrm{C}$ bottom panel (black), where bulk solution $\mathrm{pH}$ is not present at the sensing electrodes (the midpoint between protonators). With decreasing separations between the electrodes it can be observed that the generated $\mathrm{pH}$ change is becoming more uniform across the array even at this short time interval. The overlap of these individual diffusion zones after 1 second is demonstrated in Figure 5C bottom panel (red), where the variation in $\mathrm{pH}$, midpoint between protonators, is negligible. Consequently, the simulation study suggests that the $\mathrm{pH}$ has been sufficiently adjusted after only 1 second of $\mathrm{pH}$ control at these different gaps. 

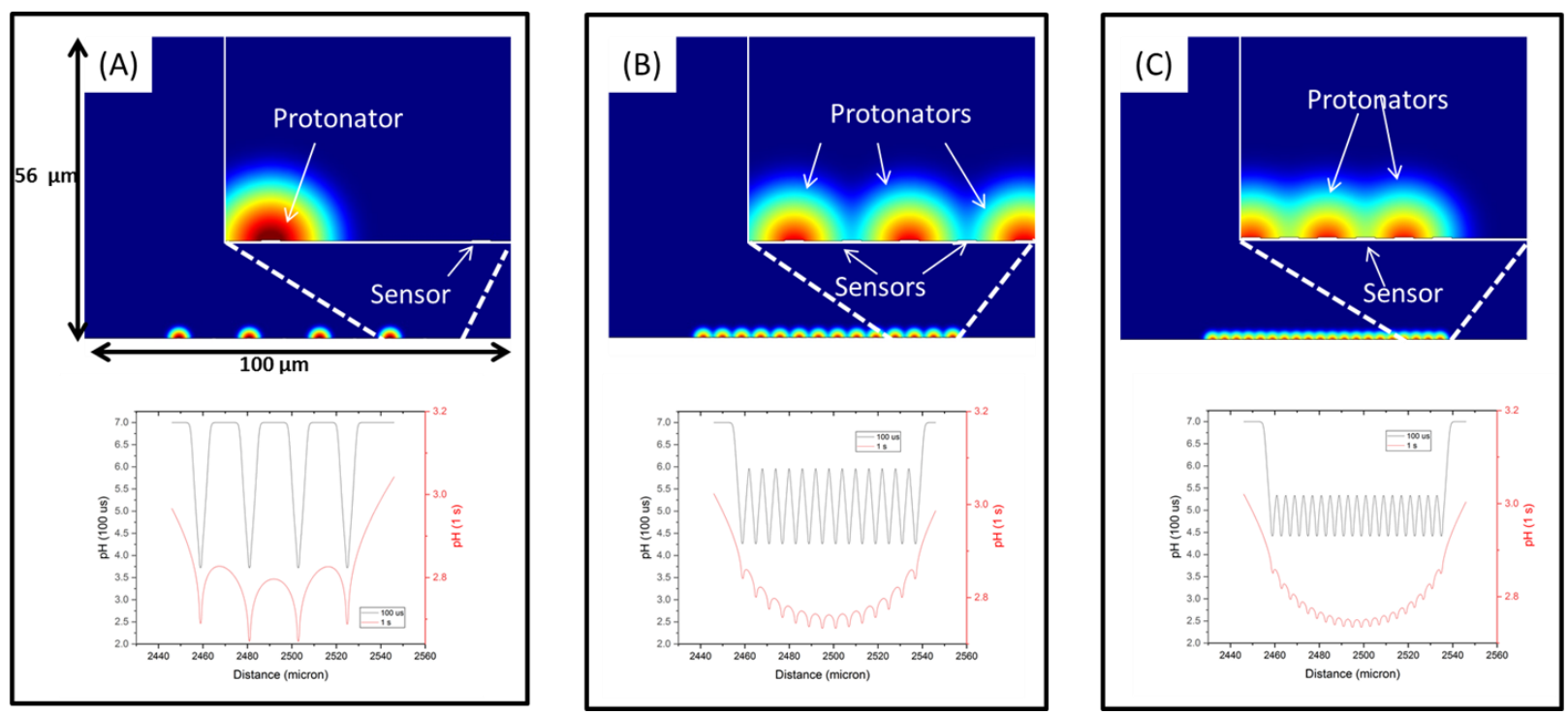

Figure 2 Top Panel: Simulated $\mathrm{pH}$ profile after $100 \mu$ s with applied current of $100 \mathrm{nA}$ at working electrode arrays with gaps of 10 (A), 2(B) and 1 (C) $\mu \mathrm{m}$. Bottom Panel (A, B, C) Plots of pH at electrode surfaces across array after $100 \mu \mathrm{s}$ and $1 \mathrm{~s}$

\subsection{Effect of initial solution $\mathbf{p H}$}

The effect of varying the initial starting $\mathrm{pH}$ of the bulk solution, over the range of $\mathrm{pH} 6$ to $\mathrm{pH} 10$, on the final $\mathrm{pH}$ was investigated. To this end, only the $2 \mu \mathrm{m}$ spacing interdigitated electrode arrays were presented as these were the electrodes that were challenged in the subsequent experimental study below. A galvanostatic current of $100 \mathrm{nA}$ was again applied to the protonators within the interdigitated electrode array. Figure 3A shows the proton concentration profile after 100 microseconds of applied current with the starting bulk pH set at $\mathrm{pH} 6$ (yellow). Individual diffusion zones observed around each of the protonators indicate the decrease in $\mathrm{pH}$ due to the flux of protons and their subsequent diffusion, highlighted by the red regions. The inset, a magnified image, shows a small degree of overlap between the diffusion zones, indicating that the local $\mathrm{pH}$ at the sensing electrodes remains relatively unchanged. Figure 3B shows the equivalent simulation when the starting $\mathrm{pH}$ was set at 10 . Again a decrease in $\mathrm{pH}$ was observed following proton production at the protonators and their subsequent diffusion. The inset shows that there is relatively more overlap of the diffusion profiles and $\mathrm{pH}$ still appears to reach $\mathrm{pH} \sim 6.0$ above the sensors. To confirm this, the final $\mathrm{pH}$ in the plane parallel to and $50 \mathrm{~nm}$ above the chip surface (i.e. at the sensor surface) is plotted in Figure $3 \mathrm{C}$ where a saw tooth $\mathrm{pH}$ profile was observed as before across the array. The peak maxima were again located over the sensors while the peak minima were over the protonators, as expected. It was observed that regardless of the initial solution $\mathrm{pH}$, a final $\mathrm{pH}$ of $\sim 6.0$ was achieved (pH 5.7 for starting $\mathrm{pH} 6$ was reached while a final $\mathrm{pH}$ of 6 was reached for starting $\mathrm{pH} 7,9$ and 10, respectively). The $\mathrm{pH}$ at the protonator surface was always observed to 
reach $\mathrm{pH} 4.2$ for different starting $\mathrm{pH}$ values. Clearly, after $100 \mu$ s there is a change of $\sim 1.7 \mathrm{pH}$ units of the solution between the protonators and the sensors. Figure 3D shows the equivalent simulation conditions after $1 \mathrm{~s}$ of applied current. Here it was observed that a final $\mathrm{pH}$, with little spatial variation, of $\sim 2.8$ was observed across the entire array. The inset shows a less magnified view to highlight initial starting $\mathrm{pH}$ of the simulations. Consequently, these results indicate that the starting $\mathrm{pH}$ does not have a significant effect on achieving the final desired $\mathrm{pH}$ at the sensors within one second of applied protonator current.

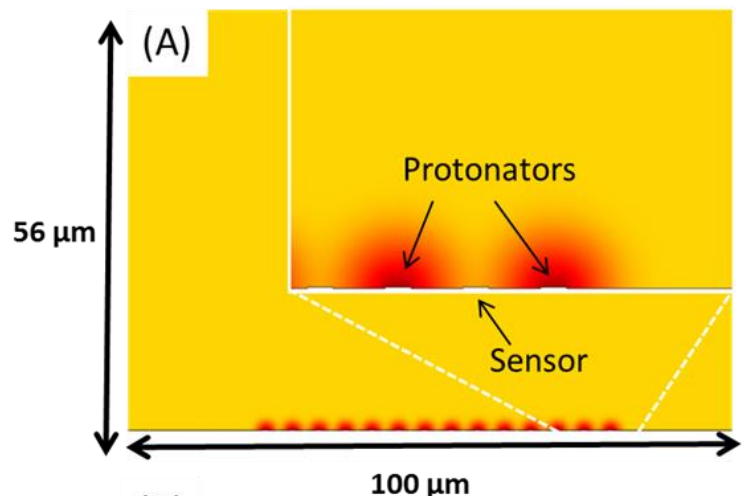

(C)

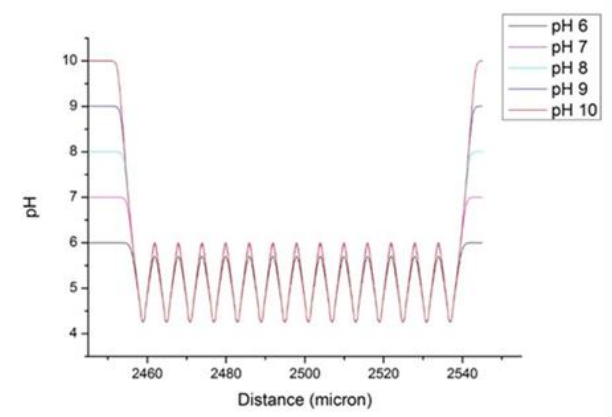

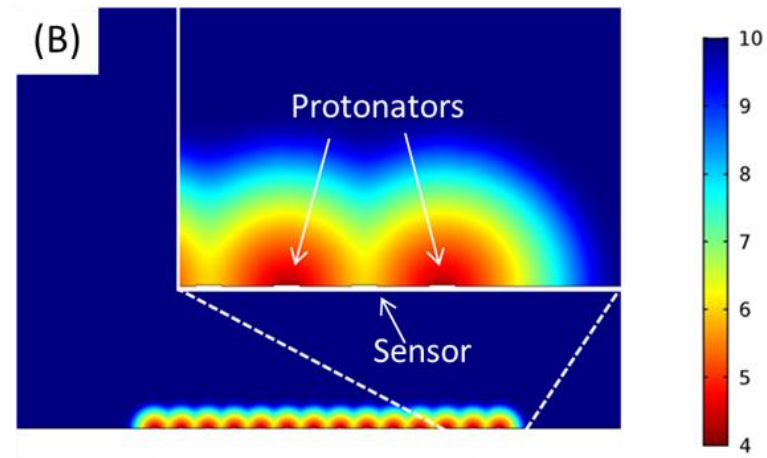

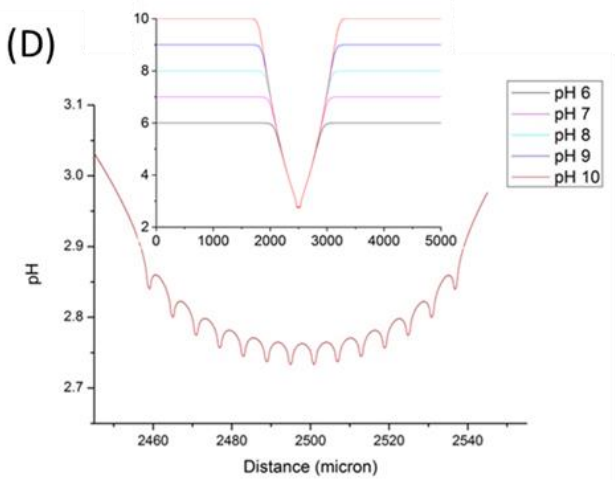

Figure 3 Simulated $\mathrm{pH}$ profile after 100 microseconds with applied current of $100 \mathrm{nA}$ at $2 \mu \mathrm{m}$ gap working electrode arrays with starting bulk pH of 6 (A) and 10 (B). (C) and (D) show plots of the $\mathrm{pH}$ at the sensor electrode surface after 100 microseconds and 1 seconds

\subsection{Solution Buffer capacity}

The model employed in the previous sections was reported by Read et al[13] and based on a purely diffusion dependant model employing a galvanostatic current. To factor in the effects of proton buffering reactions, the chemical equations from the Critelli et al[14] analysis were added (equations 5 and 6) to provide a more realistic model. Figure 4 features concentration profiles focused on a $1 \mathrm{~mm} \times 1 \mathrm{~mm}$ square by the electrodes in the modelled $5 \times 5 \mathrm{~mm}$ domain.

Figure 4A top panel is the proton concentration profile for a solution of $1 \mathrm{mM}$ bicarbonate buffer after 1 second. The diameter of the region of lower $\mathrm{pH}$ is smaller than that of the non-buffered 
model. There is a sharper transition in $\mathrm{pH}$ from protonator electrodes to the bulk $\mathrm{pH}$. The $1 \mathrm{mM}$ buffer is sufficient to narrow the diameter the area of decreased $\mathrm{pH}(340 \mu \mathrm{m}$ vs. $1,150 \mu \mathrm{m}$ unbuffered), however the flux at the electrodes still reaches $\mathrm{pH} 4$ and below. Figure 4A bottom panel is the proton concentration profile for a solution with a $1 \mathrm{mM}$ buffer after 10 seconds, where the diffusion zone of changed $\mathrm{pH}$ has increased in diameter with time $(1,050 \mu \mathrm{m}$ compared to 3,600 $\mu \mathrm{m}$ for unbuffered).

Figure 4B top panel is the proton concentration profile for a solution with a $10 \mathrm{mM}$ buffer after 1 second. The diameter of the region of lower $\mathrm{pH}$ is again reduced $(263 \mathrm{um})$. The $\mathrm{pH}$ at the electrodes has not reached $\mathrm{pH} 4$ after 1 second in a $10 \mathrm{mM}$ buffer. Figure $4 \mathrm{~B}$ bottom panel is the proton concentration profile for a solution with a $10 \mathrm{mM}$ buffer after 10 seconds. The diffusion zone diameter has increased (740 um) and local $\mathrm{pH}$ has now decreased below $\mathrm{pH} 4$. It was observed that the increased volume of protons over the 9 second interval has surpassed the buffering capacity of the buffer.

Figure 4C top panel is the proton concentration profile for a solution with a $100 \mathrm{mM}$ buffer after 1 second. The colour change indicating a lower $\mathrm{pH}$ is barely evident (184 $\mu \mathrm{m}$ with a lowest $\mathrm{pH}$ of 6.57). $100 \mathrm{mM}$ buffer significantly inhibits the electrochemical $\mathrm{pH}$ control for this applied current and duration. Figure 4C bottom panel is the proton concentration profile for a solution with a 100 $\mathrm{mM}$ buffer after 10 seconds. There is a negligible decrease in $\mathrm{pH}$ after the 9 seconds interval, due to the buffering capacity of the $100 \mathrm{mM}$ solution being greater than the flux of protons.

Figure 4D top panel is a plot of $\mathrm{pH}$ at the electrode surfaces after 1 second. For $1 \mathrm{mM}$, the $\mathrm{pH}$ at the protonators and sensors is as homogenous as an unbuffered solution. There is a large change in $\mathrm{pH}$ between the 2 combs for $10 \mathrm{mM}$. This buffer is effective after 1 second at scavenging protons over the $2 \mu \mathrm{m}$ gap between the combs. There is a slight $\mathrm{pH}$ difference between the combs at 100 $\mathrm{mM}$. This difference being smaller than that of the $10 \mathrm{mM}$ is due to the smaller relative change in $\mathrm{pH}$. Figure 4D bottom panel is a plot of $\mathrm{pH}$ at the electrode surfaces after 10 seconds. Both the 1 and $10 \mathrm{mM}$ buffer solutions now have the homogeneity of local $\mathrm{pH}$ at both electrode combs, with less of a decrease for the $10 \mathrm{mM}$ buffer. For the $100 \mathrm{mM}$, buffer the same scenario exists after 10 seconds albeit at a slightly lower $\mathrm{pH}$ (6.40). These results would suggest that to decrease the $\mathrm{pH}$ in high buffer concentrations would require a much higher current, which will be the subject of future work. 

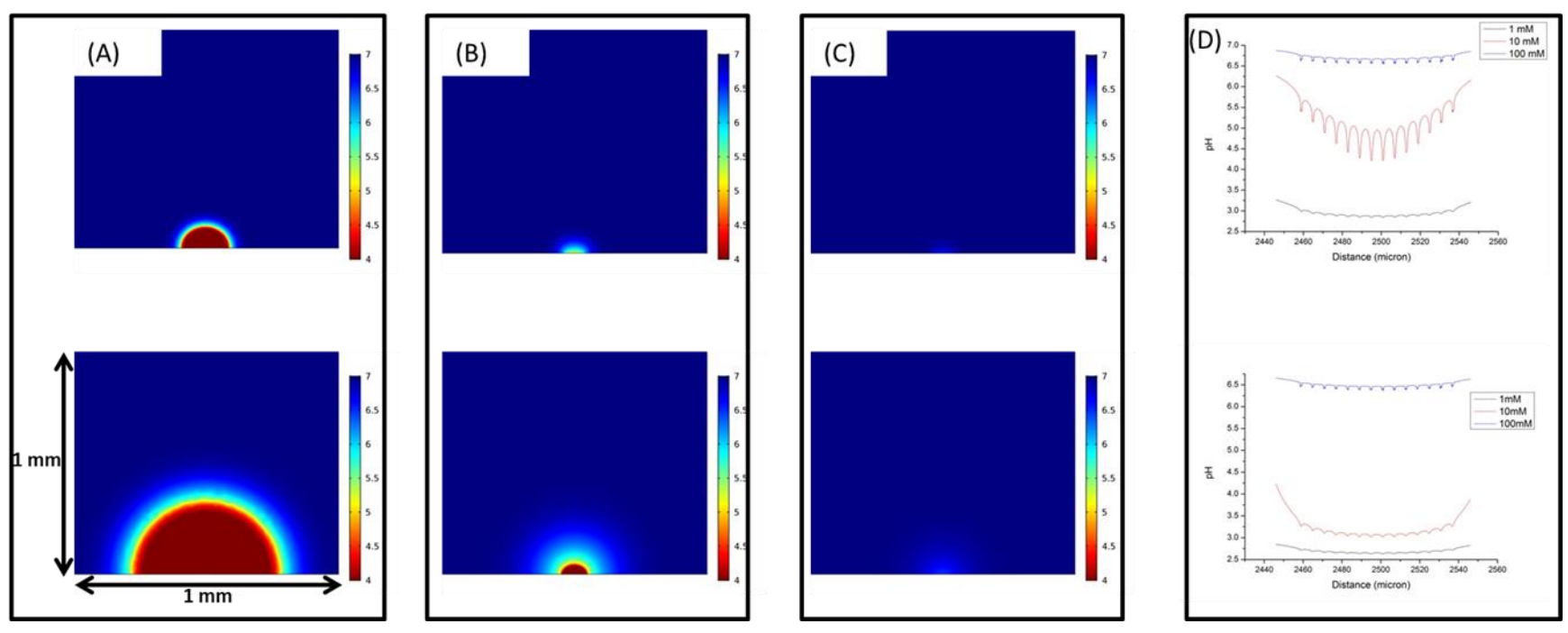

Figure 4 Top Panel: Simulated $\mathrm{pH}$ profile after 1 second with applied current of $100 \mathrm{nA}$ with buffer concentration of 1 (A), 10 (B) and 100 (C) $\mathrm{mM}$ with (D) showing a plot of $\mathrm{pH}$ across the electrode surfaces; Bottom Panel: Simulated pH profile after 10 seconds with

\subsection{Electrode Characterisation}

Experiments were undertaken, using silicon chip substrates, to confirm the simulation study presented above. Interdigitated microband electrodes were fabricated as described in the experimental section. Figure S3A shows a typical voltammogram in PBS of $\mathrm{pH} 7.4$ over the potential range of $-0.15 \mathrm{~V}$ to $1.2 \mathrm{~V}$. An increase in current beginning at $0.6 \mathrm{~V}$ indicates the onset of gold oxidation. The plateau and subsequent increase at $0.75 \mathrm{~V}$ is indicative of the formation of a complex gold oxide morphology/ multilayers. The sharp increase in current beginning at $1.1 \mathrm{~V}$ is attributed to the onset of oxygen evolution described in equations 2 or 4 . The electrochemical characterization was undertaken by cyclic voltammetry in $\mathrm{FcCOOH}$ from $-0.15 \mathrm{~V}$ to $0.45 \mathrm{~V}$ at 100 $\mathrm{mV} / \mathrm{s}$ in both three and four electrode configurations using the on-chip gold counter and platinum reference electrodes. Figure S3B presents typical voltammograms obtained. In the three electrode configuration, each IDE sensing electrode array exhibited a current magnitude of $\sim 10 \mathrm{nA}$, typical of such an array. The magnitude of this current strongly suggests that oxidation of FcCOOH only occurs at the interdigitated electrodes and that the interconnection metallization is completely passivated by the silicon nitride passivation layer. The diffusion limited peak shape arose from the electrodes of the array not being diffusionally independent due to their close proximity, as expected[12]. By contrast, in four electrode GC mode, a constant potential of $-0.15 \mathrm{~V}$ was applied at the protonator electrode while the sensor electrode was scanned. A current of $28 \mathrm{nA}$ was obtained at the sensing array electrodes with steady-state sigmoidal CV profiles observed. The increase in current arose from redox cycling of the $\mathrm{FcCOOH}$ between the protonator and sensor electrodes enabling the sensors to operate as diffusionally independent electrodes. The steady- 
state behavior again supports the thesis that the interconnection tracks were fully passivated and that redox reactions were only occurring at the IDEs.

\subsection{Gold Oxide Reduction and pH Control}

Gold oxide was formed on the sensor electrodes by sweeping the potential positively above $1.0 \mathrm{~V}$ (vs. $\mathrm{Ag} / \mathrm{AgCl}$ ). The potential of the subsequent gold oxide reduction was used to monitor solution $\mathrm{pH}$ at the microband electrodes as this potential is known to be $\mathrm{pH}$ sensitive[4]. Figure 5A shows an overlay of the gold oxide reduction peaks obtained at a sensor IDE immersed in different buffer solutions over the $\mathrm{pH}$ range 3 to 8 . The sensor IDE was scanned from -0.2 to $1.2 \mathrm{~V}$. The reduction potential of the gold oxide was observed to increase as the $\mathrm{pH}$ decreased. The change in reduction potential was then plotted versus $\mathrm{pH}$ and presented in Figure 5B. The plot exhibited excellent linearity with an $\mathrm{R}^{2}=0.994$ and a super Nernstian response of $-72.4 \mathrm{mV} / \mathrm{pH}$., clearly indicating that gold oxide potential may be used as a method of $\mathrm{pH}$ monitoring over the time scale of these experiments. A possible reason for the super-Nernstian response is the presence of different oxidation states of gold, as shown with other metals oxide such as ruthenium and iridium when used as $\mathrm{pH}$ probes.[19] We note for longer timescales, dissolution of gold from the electrode could limit this approach.

Figure 5C shows an overlay of the gold oxide reduction peak obtained at a sensor electrode using artificial drinking water solution. The sensor IDE was again scanned from -0.2 to $1.2 \mathrm{~V}$ with the protonator electrode array biased at a constant potential. A variety of different potentials ranging from 1.3 to $1.55 \mathrm{~V}$ were applied to the protonator and the reduction potential measured. An increase in the reduction potential was observed as the applied potential is increased suggesting that the $\mathrm{pH}$ is decreasing as expected. This reduction peak potential was again plotted against $\mathrm{pH}$, presented in figure 5D and again exhibited very good linearity with $\mathrm{R}^{2}=0.993$ over the timescale of the experiment. It may be concluded from these results confirm the simulation study; that the application of a suitable potential to the protonator can cause a change in solution $\mathrm{pH}$ (measured at the sensor electrodes), and that applying a greater potential leads to a larger change in $\mathrm{pH}$, as a greater flux of protons are produced. 

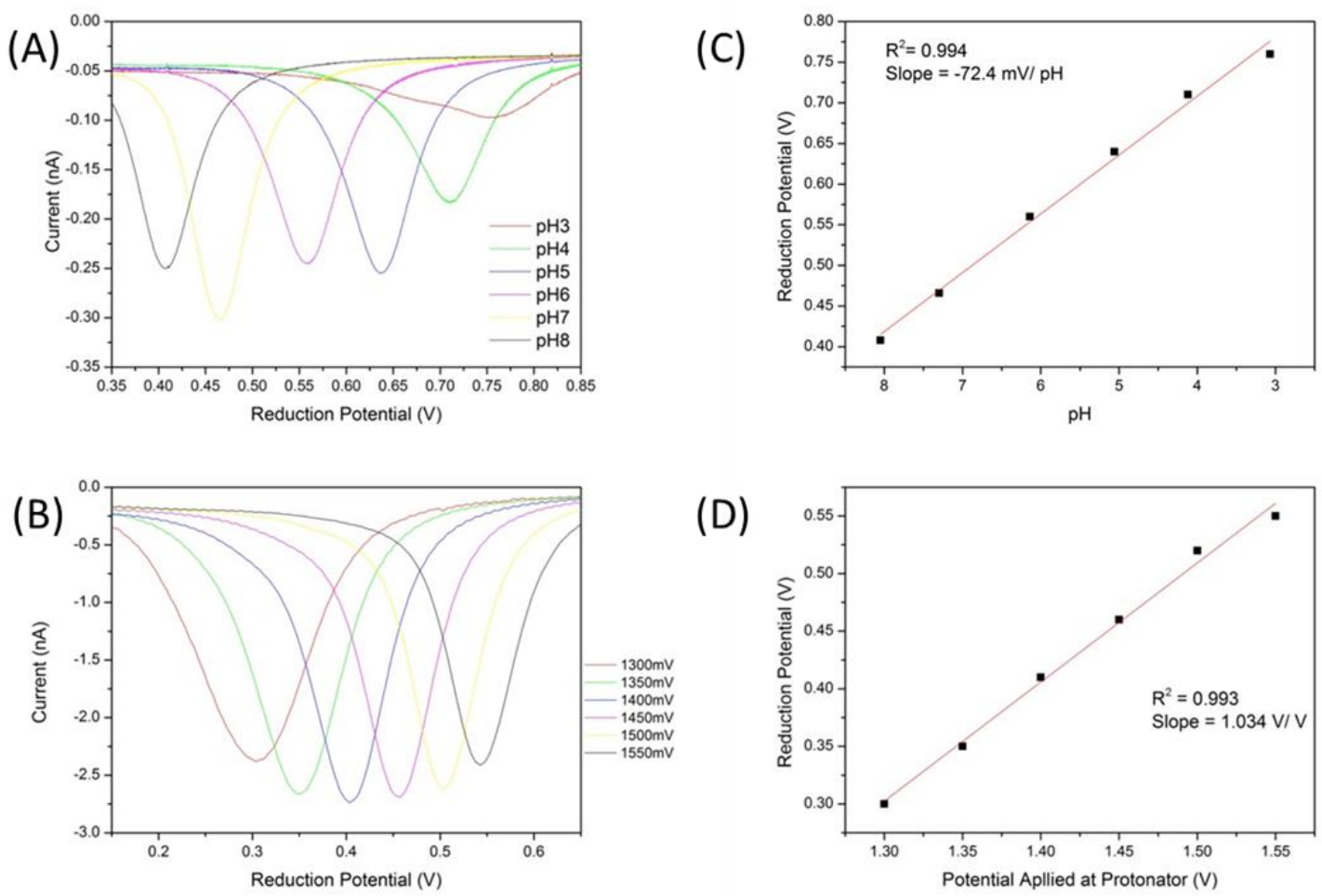

Figure 5 Reduction peak of gold oxide in chemically controlled $\mathrm{pH}$ buffers (A) with calibration plot of reduction potential vs $\mathrm{pH}(\mathrm{B})$; Reduction peak of gold oxide in electrochemically $\mathrm{pH}$ controlled buffer (C) with calibration plot of reduction potential vs applied potential(D).

\subsection{Visualization of Electrochemical $\mathrm{pH}$ control}

To further confirm the simulation study, electrochemically induced $\mathrm{pH}$ change was visualized experimentally by addition of methyl red, a $\mathrm{pH}$ indicator with a $\mathrm{pH}$ range of 4.2 (red) to 6.2 (yellow), to a $1 \mathrm{mM}$ solution of $\mathrm{ADW}$. The change of solution $\mathrm{pH}$ was monitored by use of a portable potentiostat and a microscope equipped with a $\mathrm{CCD}$, as described in the experimental section. The simulated concentration profiles of a $1 \mathrm{mM}$ buffered solution were compared to the results obtained experimentally. Figure $6 \mathrm{~A}$ top panel shows the simulated concentration profile at $\mathrm{t}=0$ seconds, while Figure $6 \mathrm{~A}$ bottom panel is an optical image of the electrodes $\mathrm{t}=0$. In both images, there is no change in colour as no current has been applied and consequently no change in $\mathrm{pH}$ has taken place. Figure $6 \mathrm{~B}$ top panel shows the simulated concentration profile at $\mathrm{t}=1 \mathrm{~s}$, while Figure 6B bottom panel is the corresponding optical image of the electrodes. A colour change is visible around the electrodes in both the simulated profile and the optical image at this time. The optical colour change arises from the generated protons interacting with the methyl red in the solution, with the colour change from yellow to red indicating that the $\mathrm{pH}$ has decreased to $\mathrm{pH} 4.2$ or below. The simulated region below $\mathrm{pH}$ has a diameter of $\sim 180 \mathrm{um}$, while the red area in the optical image has a diameter of $\sim 175 \mathrm{um}$. Figure $6 \mathrm{C}$ top panel shows the simulated concentration 
profile at $\mathrm{t}=10 \mathrm{~s}$, while Figure $6 \mathrm{C}$ bottom panel is the corresponding experimental optical image of the electrodes. The diameter of the colour change has increased in both the simulated and experimental results to $530 \mu \mathrm{m}$ and $\sim 450 \mu \mathrm{m}$, respectively. There is a slight discrepancy between the simulation's diffusion zone and the colour change visualized experimentally. There are a few potential sources of this: The ethanol added to dissolve the methyl red will change the diffusion coefficient of protons in a water based solution. The visualization technique requires a certain colour intensity to be visible. However, it is possible that protons have diffused beyond this visible region, but the colour is not intense enough to be yet observed. The addition of methyl red may also affect the diffusion coefficient of protons in solution. It has been reported by Xu et al.[20] that proton diffusion can be suppressed in solutions containing tetra methyl urea, an amphiphile with functional group similarity to methyl red.
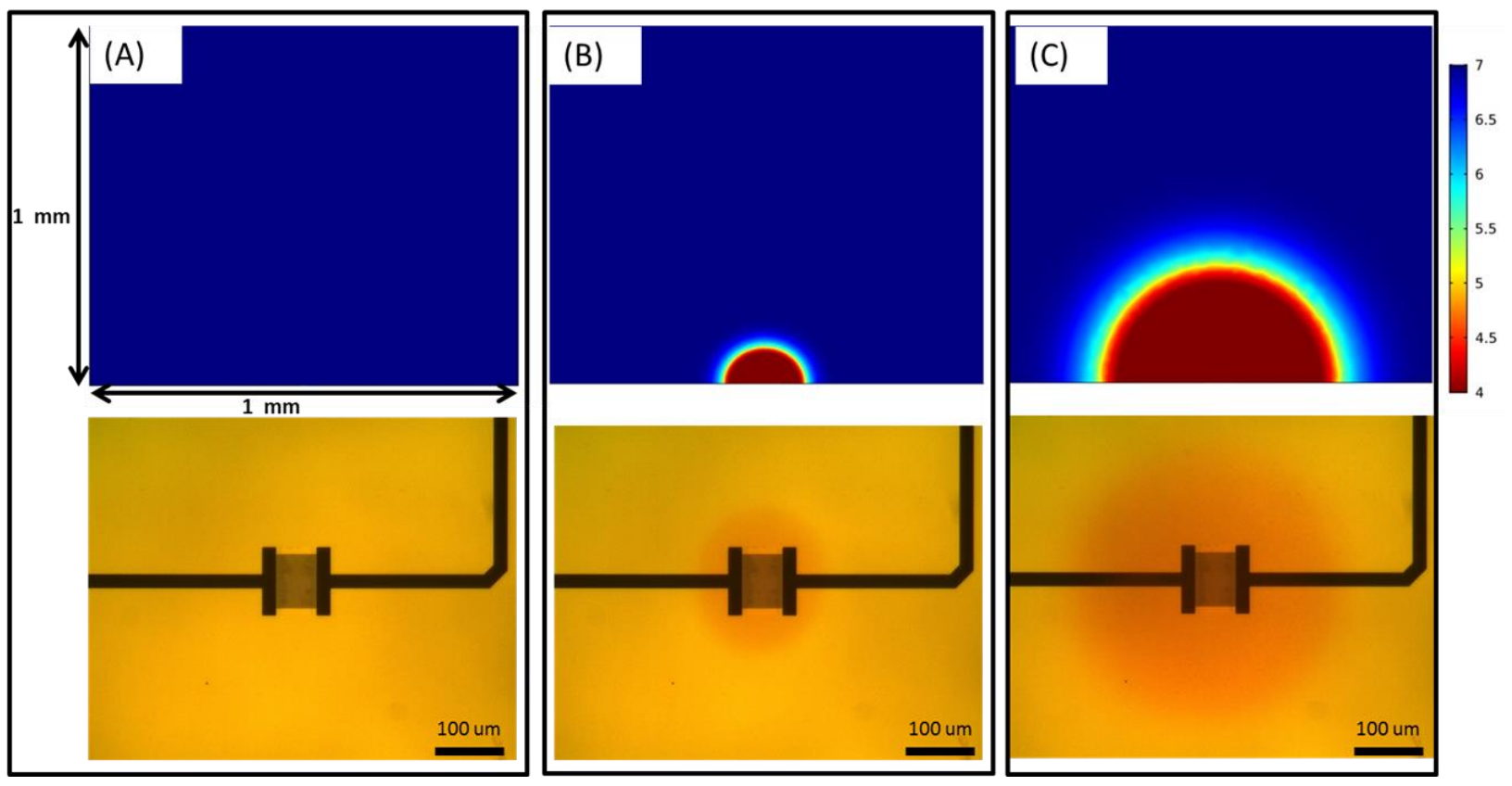

Figure 6 Top panel: Simulated $\mathrm{pH}$ profile of proton generation at protonator electrodes at 0 (A), 1 (B) and 10 (C) seconds in $1 \mathrm{mM}$ buffer; Bottom panel: Optical images of working electrode array as current applied at protonators at 0 (A), 1 (B) and 10 (C) seconds

\section{Conclusion}

We have shown a simulation and experimental study of electrochemical $\mathrm{pH}$ control at interdigitated gold microband arrays. The application of a galvanostatic current at the protonator array generates protons by water electrolysis, which diffuse away and reduce the local solution 
$\mathrm{pH}$. This method allows the $\mathrm{pH}$ at a sensor array to be adjusted while eliminating the traditional need for the skilled handling of dangerous chemicals or the inherent expense and complexity thereof. The model has highlighted the impact of the spacing between the interdigitated electrodes, where the desired $\mathrm{pH}$ is reached at the sensing electrodes more rapidly at smaller distances. The initial bulk $\mathrm{pH}$ was shown by the simulation to be unimportant in achieving the final $\mathrm{pH}$ at the electrode surfaces. Buffering reactions were factored into the model, with the buffer concentration having a clear impact on the diffusion of protons from the protonator electrodes to the sensors. Microband electrodes were fabricated in line with the geometries modelled and the of the gold oxide reduction peak was used as a measurement probe for the local solution $\mathrm{pH}$ at the surface of the electrodes. This work shows the feasibility of using a $\mathrm{pH}$ indicator to corroborate the region of $\mathrm{pH}$ change around the protonator and sensing electrodes predicted by FEA. This in turn supports the use of electrochemical $\mathrm{pH}$ control in electroanalysis, where the analytes of interest are $\mathrm{pH}$ dependent.

\section{Acknowledgements}

The authors would like to acknowledge funding from the Irish Research Council (Project GOIPG/2015/2925). This work has been supported in part by a research grant for the VistaMilk Centre Science Foundation Ireland (SFI); Department of Agriculture, Food and the Marine (DAFM) under Grant Number 16/RC/3835

\section{References}

[1] T.W. Kaaret, G.H. Zhang, T.C. Bruice, Electrochemistry and the dependence of potentials on pH of iron and manganese tetraphenylporphyrins in aqueous solution, Journal of the American Chemical Society, 113(1991) 4652-6.

[2] J. Davis, M.J. Moorcroft, S.J. Wilkins, R.G. Compton, M.F. Cardosi, Electrochemical detection of nitrate and nitrite at a copper modified electrode, The Analyst, 125(2000) 737-42.

[3] B. Patella, R.R. Russo, A. O'Riordan, G. Aiello, C. Sunseri, R. Inguanta, Copper nanowire array as highly selective electrochemical sensor of nitrate ions in water, Talanta, 221(2021) 121643.

[4] I. Seymour, B. O'Sullivan, P. Lovera, J.F. Rohan, A. O'Riordan, Electrochemical detection of freechlorine in Water samples facilitated by in-situ $\mathrm{pH}$ control using interdigitated microelectrodes, Sensors and Actuators B: Chemical, 325(2020) 128774.

[5] R.J. Toh, W.K. Peng, J. Han, M. Pumera, Haemoglobin electrochemical detection on various reduced graphene surfaces: well-defined glassy carbon electrode outperforms the graphenoids, RSC Advances, 4(2014) 8050-4.

[6] G.A.S. Minero, P.F. Wagler, A.A. Oughli, J.S. McCaskill, Electronic pH switching of DNA triplex reactions, RSC Advances, 5(2015) 27313-25.

[7] P.A. Michaud, M. Panizza, L. Ouattara, T. Diaco, G. Foti, C. Comninellis, Electrochemical oxidation of water on synthetic boron-doped diamond thin film anodes, Journal of Applied Electrochemistry, 33(2003) 151-4.

[8] A. Wahl, K. Dawson, J. MacHale, S. Barry, A.J. Quinn, A. O'Riordan, Gold nanowire electrodes in array: simulation study and experiments, Faraday Discussions, 164(2013) 377-90. 
[9] N. Godino, X. Borrisé, F.X. Muñoz, F.J. del Campo, R.G. Compton, Mass Transport to Nanoelectrode Arrays and Limitations of the Diffusion Domain Approach: Theory and Experiment, The Journal of Physical Chemistry C, 113(2009) 11119-25.

[10] D.W.M. Arrigan, G. Herzog, Theory of electrochemistry at miniaturised interfaces between two immiscible electrolyte solutions, Current Opinion in Electrochemistry, 1(2017) 66-72.

[11] M. Yang, R.G. Compton, Adsorption processes coupled with mass transport at macro-electrodes: New insights from simulation, Journal of Electroanalytical Chemistry, 836(2019) 68-76.

[12] A.J.C. Wahl, I.P. Seymour, M. Moore, P. Lovera, A. O'Riordan, J.F. Rohan, Diffusion profile simulations and enhanced iron sensing in generator-collector mode at interdigitated nanowire electrode arrays, Electrochimica Acta, 277(2018) 235-43.

[13] T.L. Read, E. Bitziou, M.B. Joseph, J.V. Macpherson, In Situ Control of Local pH Using a Boron Doped Diamond Ring Disk Electrode: Optimizing Heavy Metal (Mercury) Detection, Analytical Chemistry, 86(2014) 367-71.

[14] R.A.J. Critelli, M. Bertotti, R.M. Torresi, Probe effects on concentration profiles in the diffusion layer: Computational modeling and near-surface $\mathrm{pH}$ measurements using microelectrodes, Electrochimica Acta, 292(2018) 511-21.

[15] T.L. Read, M.B. Joseph, J.V. Macpherson, Manipulation and measurement of $\mathrm{pH}$ sensitive metalligand binding using electrochemical proton generation and metal detection, Chemical Communications, 52(2016) 1863-6.

[16] J. Rossmeisl, A. Logadottir, J.K. Nørskov, Electrolysis of water on (oxidized) metal surfaces, Chemical Physics, 319(2005) 178-84.

[17] S.H. Lee, J.C. Rasaiah, Proton transfer and the mobilities of the $\mathrm{H}+$ and $\mathrm{OH}-$ ions from studies of a dissociating model for water, The Journal of Chemical Physics, 135(2011) 124505.

[18] A. Montrose, N. Creedon, R. Sayers, S. Barry, A. O'Riordan, Novel Single Gold Nanowire-based Electrochemical Immunosensor for Rapid Detection of Bovine Viral Diarrhoea Antibodies in Serum, Journal of Biosensors \& Bioelectronics, 6(2015).

[19] K. Singh, B.-S. Lou, J.-L. Her, S.-T. Pang, T.-M. Pan, Super Nernstian pH response and enzyme-free detection of glucose using sol-gel derived RuOx on PET flexible-based extended-gate field-effect transistor, Sensors and Actuators B: Chemical, 298(2019) 126837.

[20] J. Xu, T. Yamashita, N. Agmon, G.A. Voth, On the Origin of Proton Mobility Suppression in Aqueous Solutions of Amphiphiles, The Journal of Physical Chemistry B, 117(2013) 15426-35. 